\title{
Chlamydomonas reinhardtii thermal tolerance enhancement mediated by a mutualistic interaction with vitamin $\mathrm{B}_{12}$-producing bacteria
}

\author{
Bo Xie $^{1}$, Shawn Bishop ${ }^{2}$, Dan Stessman ${ }^{2}$, David Wright ${ }^{2}$, Martin H Spalding ${ }^{2}$ and \\ Larry J Halverson ${ }^{1}$ \\ ${ }^{1}$ Department of Plant Pathology and Microbiology, Iowa State University, Ames, IA, USA and ${ }^{2}$ Departments of \\ Genetics, Development and Cell Biology, Iowa State University, Ames, IA, USA
}

\begin{abstract}
Temperature is one of the most important environmental factors affecting the growth and survival of microorganisms and in light of current global patterns is of particular interest. Here, we highlight studies revealing how vitamin $B_{12}$ (cobalamin)-producing bacteria increase the fitness of the unicellular alga Chlamydomonas reinhardtii following an increase in environmental temperature. Heat stress represses $C$. reinhardtii cobalamin-independent methionine synthase (METE) gene expression coinciding with a reduction in METE-mediated methionine synthase activity, chlorosis and cell death during heat stress. However, in the presence of cobalamin-producing bacteria or exogenous cobalamin amendments $C$. reinhardtii cobalamin-dependent methionine synthase METHmediated methionine biosynthesis is functional at temperatures that result in $C$. reinhardtii death in the absence of cobalamin. Artificial microRNA silencing of $C$. reinhardtii METH expression leads to nearly complete loss of cobalamin-mediated enhancement of thermal tolerance. This suggests that methionine biosynthesis is an essential cellular mechanism for adaptation by $C$. reinhardtii to thermal stress. Increased fitness advantage of METH under environmentally stressful conditions could explain the selective pressure for retaining the METH gene in algae and the apparent independent loss of the METE gene in various algal species. Our results show that how an organism acclimates to a change in its abiotic environment depends critically on co-occurring species, the nature of that interaction, and how those species interactions evolve.
\end{abstract}

The ISME Journal (2013) 7, 1544-1555; doi:10.1038/ismej.2013.43; published online 14 March 2013

Subject Category: Microbe-microbe and microbe-host interactions

Keywords: bacteria-algae interactions; cobalamin-independent methionine synthase; cobalamindependent methionine synthase; gene regulation; stress tolerance; vitamin $\mathrm{B}_{12}$

\section{Introduction}

There has been increasing interest in the interactions between bacteria, algae and their environment, and how those interactions influence alga morphogenesis, nutrient acquisition and community composition as well as bacterial growth and biogeochemical cycles (for example, Croft et al., 2005; Matsuo et al., 2005; Matz et al., 2008; Amin et al., 2009; Barott et al., 2011; Seyedsayamdost et al., 2011). Understanding how these interactions influence the adaption of bacteria and algae to the abiotic environment is important for understanding the physiological processes and evolutionary outcomes of those species as nearly all organisms cooccur with other species. In the present study, we

Correspondence: LJ Halverson, Department of Plant Pathology and Microbiology, Iowa State University, 304 Science I, Ames, IA 50010, USA.

E-mail: larryh@iastate.edu

Received 17 September 2012; revised 6 February 2013; accepted 11 February 2013; published online 14 March 2013 identified how a beneficial interaction between certain bacterial species and the alga Chlamydomonas reinhardtii influences the ability of $C$. reinhardtii to survive a change in its abiotic environment that is lethal to $C$. reinhardtii in isolation.

C. reinhardtii, a unicellular eukaryotic microalga found in soil and aquatic environments, grows with $\mathrm{CO}_{2}$ and light, and is considered mesophilic (20-32 ${ }^{\circ} \mathrm{C}$ optimum). An abrupt transfer to a temperature above the typical growth range (for example, $39^{\circ} \mathrm{C}$ ) results in heat shock, triggering adaptive responses that may or may not allow resumption of growth or survival at the new temperature (Schroda, 2004). Prolonged exposure to incompatible temperatures results in a cessation of metabolism, chlorosis and ultimately cell death. Acclimation to high temperature relies on basal mechanisms to initiate physiological adjustments that permit expression of adaptive mechanisms required for prolonged survival at those and higher temperatures. Similar to other eukaryotes, heat shock transcription factor and heat shock proteins 
that facilitate proper protein folding and turnover are highly induced upon heat treatment in $C$. reinhardtii (Schulz-Raffelt et al., 2007).

Many algal species require exogenous cobalamin (vitamin $\mathrm{B}_{12}$ ), thiamine (vitamin $\mathrm{B}_{1}$ ) or biotin (vitamin $\mathrm{B}_{7}$ ) for growth (Croft et al., 2006). As cobalamin is produced only by prokaryotes (Roth et al., 1996; Warren et al., 2002), cobalamin could be acquired by scavenging it from the environment or through a mutualistic or commensal association with cobalamin-producing bacteria (Croft et al., 2005, 2006; Kazamia et al., 2012). Cobalamin is a $\mathrm{Co}^{2+}$-containing, modified tetrapyrrole that functions as a cofactor for enzymes involved in C1 and other metabolic reactions (Roth et al., 1996). In many eukaryotic algae (Croft et al., 2005; Helliwell et al., 2011), cobalamin requirements arise primarily from its use by methionine synthase, responsible for synthesizing methionine and tetrahydrofolate from homocysteine and 5-methyl-tetrahydrofolate (Roth et al., 1996; Warren et al., 2002). C. reinhardtii has only one known cobalamin-dependent enzyme, methionine synthase (METH), and the accessory proteins methionine synthase reductase and AdoCbl synthase required for S-adenosylhomocysteine reduction and adenosylcobalamin synthesis, respectively (Croft et al., 2005; Helliwell et al., 2011). Yet, C. reinhardtii also has a cobalaminindependent methionine synthase (METE) (Croft et al., 2005; Helliwell et al., 2011) that accomplishes the same reaction as METH albeit likely with lower efficiency (Gonzalez et al., 1992). Aside from avoiding dependency on cobalamin for methionine synthesis and the greater ecological flexibility it provides in environments with scarce cobalamin availability, little is known of the advantages or disadvantages of one isoform of methionine synthase over the other in contributing to the ecological success of $C$. reinhardtii or other algae in natural habitats.

In the course of another study, we observed that C. reinhardtii thermal tolerance was enhanced when cultures were contaminated with bacteria, which led to a series of experiments to elucidate the mechanism of this enhancement. Our data show that certain cobalamin-producing bacterial species when co-cultivated with $C$. reinhardtii enhance algal thermal tolerance, and that cobalamin-dependent methionine biosynthesis has a critical role in thermal tolerance enhancement. These findings will have implications for better understanding ecosystem functioning as marine algae contribute substantially to primary productivity (Field et al., 1998; Falkowski et al., 2004), and are therefore important factors in global carbon cycling. Consequently, it might be appropriate to include cobalamin availability and cobalaminproducing bacteria and their production and consumption dynamics in efforts to predict and model marine or terrestrial primary productivity as temperatures rise.

\section{Materials and methods}

C. reinhardtii and bacterial strains and growth conditions

Wild-type C. reinhardtii strains 21gr (cc1690) and 137c (cc125) were obtained from the Chlamydomonas Resource Center (http://chlamycollection.org/) and were grown phototrophically under continuous illumination $\left(120 \mu \mathrm{E} \mathrm{m}^{-2} \mathrm{sec}^{-1}\right)$ in minimal salts medium or on agar $(1.5 \%)$ solidified plates (Geraghty et al., 1990) in a $5 \% \mathrm{CO}_{2}$ atmosphere. Bacterial species were grown on TY (Beringer 1974) or rhizobial minimal medium (Broughton et al., 1986) at 25 or $30^{\circ} \mathrm{C}$. Sinorhizobium meliloti wildtype 1021 and its Tn5 transposon insertion mutants $\mathrm{cobD}^{-}$, bluB ${ }^{-}$and $\mathrm{metH}^{-}$(Campbell et al., 2006; Taga et al., 2007) were generously provided by Michiko Taga (University of California-Berkeley). The cobalamin-deficient $c o b D^{-}$mutant was grown in TY media supplemented with $2 \mathrm{mg} \mathrm{l}^{-1}$ cyanocobalamin. Algal growth media were supplemented with cyanocobalamin (Cbl), methionine (Met), thiamine, biotin or folate at the indicated concentrations. S. meliloti growth media were supplemented with $25 \mu \mathrm{g} \mathrm{ml}^{-1}$ kanamycin or $20 \mu \mathrm{g} \mathrm{ml}^{-1}$ gentamycin. Culture supernatant was obtained by centrifugation of a 3-day-old stationary phase $S$. meliloti culture grown in rhizobial minimal medium, and decanting the culture supernatant, filter sterilizing it, drying it in a speed-vac and re-suspending it in water. The identity of air-borne bacterial contaminants of Chlamydomonas cultures that exhibited thermal tolerance enhancing properties was based on sequencing of the 16S rRNA gene (see Supplementary Information for details).

\section{Thermal tolerance assay}

Our thermal tolerance plate assays consisted of growing Chlamydomonas for $0-3$ days at $25^{\circ} \mathrm{C}$ in the presence or absence of bacteria, or cobalamin or methionine amendments to the culture media before transferring plates (upshift) to higher temperatures. Enhancement of thermal tolerance is defined as a delay in chlorosis at normally lethal temperatures and was assessed 5 days post temperature upshift. Chlamydomonas liquid cultures were adjusted to $1.5 \times 10^{6}$ cell per ml with fresh medium, based on direct microscopy counts. Sinorhizobium were washed twice and re-suspended from plate-grown cultures in a $0.85 \%$ saline solution, and serially diluted in a saline solution before mixing equal volumes of bacteria and $C$. reinhardtii cell suspensions to generate varying inoculum ratios; cell densities were verified by determining colony forming units. These mixtures were inoculated onto plates as co-cultures comprised of the same number of Chlamydomonas cells (5000, unless stated otherwise), but the abundance of $S$. meliloti cells varied. To minimize cyanocobalamin contamination from the medium, the $S$. meliloti $c o b D^{-}$mutant was 
sub-cultured for 1.5 days in TY broth before preparing cells for co-inoculation as described above. Alternatively, $C$. reinhardtii was streaked onto minimal salts medium plates before inoculating selected areas with bacteria by streaking. For determining viable cell numbers, co-cultures were removed from the plate and serially diluted before plating aliquots onto minimal salts medium or TY plates for enumeration of Chlamydomonas or Sinorhizobium, respectively. Cobalamin production was determined using a modified version of a previously described bioassay (Raux et al., 1996) and epifluorescence microscopy imaging of co-cultures are outlined in Supplementary Information.

Methionine synthase activity assays

Chlamydomonas cells were lysed by sonication in $0.1 \mathrm{M} \mathrm{KPO}_{4}$ buffer containing $10 \mathrm{~mm}$ DTT, pH 7.2 and $0.1 \mathrm{~mm}$ phenylmethylsulfonyl fluoride, and the mixture was centrifuged at $14000 \mathrm{~g}$ for $60 \mathrm{~min}$ at $4{ }^{\circ} \mathrm{C}$. The supernatant provided a crude enzyme prep for methionine synthase activity assays using the method described previously (Drummond et al., 1995), with modification. Protein concentrations were determined using the Bradford assay (Bio-Rad, Hercules, CA, USA) and 0.1-0.2 $\mathrm{mg} \mathrm{ml}^{-1}$ BSA-equivalents of crude enzyme prep was used in each assay. Assay substrates include $0.5 \mathrm{~mm}$ L-homocysteine (Fisher Scientific, Pittsburgh, PA, USA) and $0.16 \mathrm{~mm} 5$-methyl-tetrahydrofolate monoglutamate (Schircks Laboratories, Jona, Switzerland) for cobalamin-dependent METH or $0.16 \mathrm{~mm}$ 5methyl-tetrahydrofolate triglutamate (Schircks Laboratories) for cobalamin-independent METE activity assays. Cyanocobalamin was provided at a concentration of $0.05 \mu \mathrm{M}$ for METH activity assays. Assays were performed at $37^{\circ} \mathrm{C}$ for $45 \mathrm{~min}$ and quenched by adding a $5-\mathrm{N} \mathrm{HCl} / 60 \%$ formic acid solution. As the product tetrahydrofolate is unstable in acidic solutions, the reaction mixture was heated at $80{ }^{\circ} \mathrm{C}$ for $10 \mathrm{~min}$ to form methenyltetrahydrofolate. After centrifugation at $14000 \mathrm{~g}$ for $5 \mathrm{~min}$, the absorbance $(350 \mathrm{~nm})$ of methenyltetrahydrofolate in the reaction mixture was measured. Assay mixtures devoid of crude enzyme prep or substrates were used as blanks.

Gene silencing of METH in C. reinhardtii

Artificial miRNA oligonucleotides were designed using the Web MicroRNA Designer platform (http:// wmd3.weigelworld.org/cgi-bin/webapp.cgi). The MetHmi-1F and MetHmi-1R oligonucleotides (Supplementary Table S1) targeting C. reinhardtii METH coding sequence (439-448 bp downstream of the start nucleotide base) were annealed and ligated into SpeI-digested pChlamiRNA3int as described previously (Molnar et al., 2009) to generate the artificial microRNA (amiRNA) vector pChlamiRNA3int-METH for transformation with $C$. reinhardtii strain CC125. Transformants were selected on TAP (Gorman and Levine, 1965) solid medium containing $15 \mu \mathrm{g} \mathrm{ml}^{-1}$ paromomycin. The mRNA levels of METH were analyzed using qRT-PCR with the primers listed in Supplementary Table S1. The knockdown lines with reduced METH mRNA levels were then examined for their ability to delay chlorosis at elevated temperatures in the absence or presence of cyanocobalamin, methionine or S. mililoti 1021. Procedures for RNA isolation for RT and qRT-PCR are described in Supplementary Information.

\section{Results}

Enhancement of thermal tolerance in C. reinhardtii by bacteria

In the course of another study, we observed that C. reinhardtii exhibited delayed chlorosis at normally lethal temperatures when contaminated with bacteria. This observation lead us to examine what features of this bacterial-Chlamydomonas interaction facilitated increased fitness at elevated temperatures. The contaminating bacterial isolates and others available in our collection were co-cultured with $C$. reinhardtii to assess whether enhancement of thermal tolerance (a.k.a. delayed chlorosis) was species specific using the assay described in the Materials and methods and the footnote to Table 1 . Only bacteria of the $\alpha$-proteobacterial lineage strongly decreased $C$. reinhardtii chlorosis at elevated temperatures (Table 1, Supplementary Figure S1).

As S. meliloti 1021 (recently renamed Ensifer meliloti) most consistently enhanced Chlamydomonas thermal tolerance and this strain has been shown to benefit from its interaction with algae (Kazamia et al., 2012 and unpublished data), we used this strain to further establish the ability of bacterial interactions to delay algal chlorosis and increase survival at elevated temperatures. In these co-cultures $S$. meliloti forms aggregates between and in close association with $C$. reinhardtii cells and is not internalized by $C$. reinhardtii, based on epifluorescence microscopy (Supplementary Figure S2). Our visual assessment of Chlamydomonas chlorosis and survival at $42{ }^{\circ} \mathrm{C}$ was validated by C. reinhardtii $21 \mathrm{gr}$ chlorophyll content and viability of the co-cultures which was greatly influenced by the $S$. meliloti inoculum size (Figures 1a,d and e). Shifts in the relative abundances of $C$. reinhardtii and $S$. meliloti occurred quickly (Figure 1b) leading to a 5-9-fold greater abundance of $S$. meliloti by 8 DAI (Figure 1c) in the absence of heat stress. $C$. reinhardtii death in the $42{ }^{\circ} \mathrm{C}$ treatment does not appear to provide sufficient resources to promote $S$. meliloti growth or survival beyond the levels observed before the temperature upshift (compare Figure 1b with Figure 1d). In contrast, co-cultures with $S$. meliloti in the absence of thermal stress had 
Table 1 Specificity of bacterial enhancement of Chlamydomonas thermal tolerance ${ }^{\mathrm{a}}$

\begin{tabular}{|c|c|c|c|}
\hline Species & Phylogenetic class (family) & Thermal tolerance ${ }^{\mathrm{b}}$ & Aerobic vitamin $B_{12}$ synthesis ${ }^{\mathrm{c}}$ \\
\hline Aeromicrobium tamlense ${ }^{\mathrm{d}}$ & Actinobacteria & \pm & + \\
\hline Brevibacillus panacihumi $^{\mathrm{d}}$ & Firmicutes & + & + \\
\hline Mesorhizobium $s p^{\mathrm{d}}$ & $\alpha$-Proteobacteria (Phyllobacteriaceae) & $2+$ & + \\
\hline Mesorhizobium plurifurium ${ }^{\mathrm{d}}$ & $\alpha$-Proteobacteria (Phyllobacteriaceae) & $2+$ & + \\
\hline Roseomonas mucosa ${ }^{\mathrm{d}}$ & $\alpha$-Proteobacteria (Acetobacteriaceae) & $2+$ & + \\
\hline Sinorhizobium meliloti 1021 & $\alpha$-Proteobacteria (Rhizobiaceae) & $2+$ & + \\
\hline S. meliloti Mn04-gfp & $\alpha$-Proteobacteria (Rhizobiaceae) & $2+$ & + \\
\hline Alcaligenes faecalis & $\beta$-Proteobacteria (Alcaligenaceae) & - & + \\
\hline Burkholderia cepacia ATCC 25416 & $\beta$-Proteobacteria (Burkholdereaceae) & \pm & + \\
\hline Pseudomonas aeruginosa PA01 & $\gamma$-Proteobacteria (Pseuodomonadaceae) & - & + \\
\hline Pseudomonas putida mt2 & $\gamma$-Proteobacteria (Pseuodomonadaceae) & - & + \\
\hline Enterobacter cloacae JL1157 & $\gamma$-Proteobacteria (Enterobacteriaceae) & - & - \\
\hline Serratia marcescens & $\gamma$-Proteobacteria (Enterobacteriaceae) & - & - \\
\hline Salmonella enterica typhimurium LT2 & $\gamma$-Proteobacteria (Enterobacteriaceae) & \pm & - \\
\hline
\end{tabular}

${ }^{\text {a }} \mathrm{C}$. reinhardtii and bacterial co-cultures were grown at $25^{\circ} \mathrm{C}$ for 3 days before shifting to $42{ }^{\circ} \mathrm{C}$, a temperature that typically causes chlorosis and presumably cell death after $3-5$ additional days of incubation. Controls were kept at $25^{\circ} \mathrm{C}$.

bThermal tolerance was assessed visually as illustrated in Supplementary Figure S1. Thermal tolerance enhancing effect is defined as no ( -$)$, weak $( \pm)$, moderate $(+)$ or robust $(2+)$.

${ }^{c}$ Vitamin $B_{12}$ production was verified in aerobic cultures using a bioassay described in the Materials and methods and Supplementary Information file.

${ }^{\mathrm{d}}$ Bacterium isolated from contaminated Chlamydomonas cultures and identified by sequencing a near full-length PCR amplicon of the 16S rRNA gene.

${ }^{\text {eVitamin }} \mathrm{B}_{12}$ synthesized anaerobically (Jeter et al., 1984).

no effect on $C$. reinhardtii, based on chlorophyll content (Figures 1a and e) and survival (Figure 1c), even with large initial $S$. meliloti inoculum sizes. Ethanol-killed S. meliloti inoculated in a 100-fold excess of $C$. reinhardtii did not confer thermal protection although highly concentrated sterilized culture supernatants did (Figure 1f), indicating that $S$. meliloti secretes a metabolite(s) or the component(s) can be released upon cell death.

Enhancement of C. reinhardtii thermal tolerance by vitamin $B_{12}$ or methionine

Many algal species require cobalamin, thiamine or biotin for growth (Croft et al., 2006), and given that only some bacteria or Archaea species produce cobalamin this resource is possibly acquired through an algal-bacterial symbiosis (Croft et al., 2005; Helliwell et al., 2011). C. reinhardtii does not require cobalamin for growth as it has genes for both cobalamin-dependent (METH) and -independent (METE) methionine synthase (Croft et al., 2005; Helliwell et al., 2011). Yet, it's conceivable one or more of these vitamins could affect algal growth under high temperature. Of the vitamins or related compounds examined, only cyanocobalamin (Table 2 and Figure 2a) significantly delayed C. reinhardtii chlorosis at elevated temperatures, with a minimum requirement of $100 \mathrm{ngl}^{-1}$ (74 pMol) (Figure 2a). As cobalamin is the methyldonating cofactor of methionine synthase METH, which catalyzes the synthesis of methionine and tetrahydrofolate from homocysteine and methyltetrahydrofolate, we included methionine and folate as well. The requirement for near mм methionine concentrations (Table 2 and Figure 2) to effectively enhance $C$. reinhardtii thermal tolerance likely reflects the fortuitous uptake by transporters not specific to methionine as described earlier (Kirk and Kirk, 1978). Folate had no effect on thermal tolerance. Finally, cobalamin or methionine rescue of C. reinhardtii heat sensitivity is not restricted to specific strains, but was observed in a variety of wildtype and mutant strains (Supplementary Table S2) albeit the extent of tolerance varied among strains.

Chlamydomonas thermal tolerance was influenced by when cells were exposed to cobalamin or methionine (Figure 2b) and by the length of the acclimation period (Figure 2c) before a temperature upshift in a cell density-dependent manner (Figures $2 \mathrm{~b}$ and $\mathrm{c}$ ). In the absence of any pre-acclimation at $25{ }^{\circ} \mathrm{C}$ before a temperature upshift, planktonic cultures grown in the presence of cobalamin or methionine required higher cell densities to exhibit, albeit weak, enhanced thermal tolerance (Figure 2b). In a cell-dependent manner, the longer the acclimation time at $25^{\circ} \mathrm{C}$ the better the thermal tolerance (Figure 2c). In contrast, methionine-mediated enhancement of thermal tolerance occurred only after a prolonged (3-day) acclimation period before the temperature upshift (Figure 2c). Cyanocobalamin and to a lesser extent methionine could alleviate heat sensitivity up to $44^{\circ} \mathrm{C}$, but had no effect at higher temperatures (Supplementary Table S3). Cyanocobalamin rescue of chlorosis and heat sensitivity did not rely on large temperature upshifts (for example, $25-42^{\circ} \mathrm{C}$ ), as we observed similar thermal tolerance enhancement with temperature upshifts from 38 to $42{ }^{\circ} \mathrm{C}$, although chlorosis occurred more slowly in the absence of cyanocobalamin presumably owing to heat-induced metabolic adjustments or reduced metabolic activity of cells 


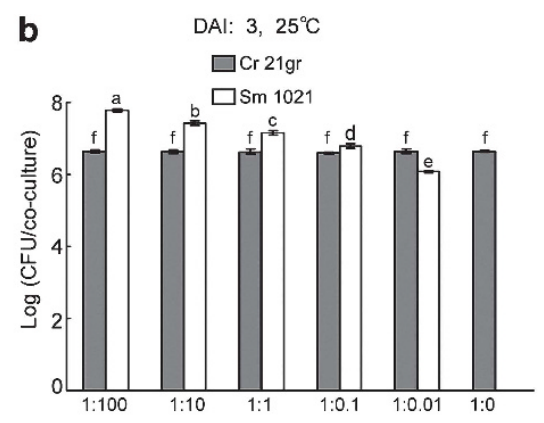

C
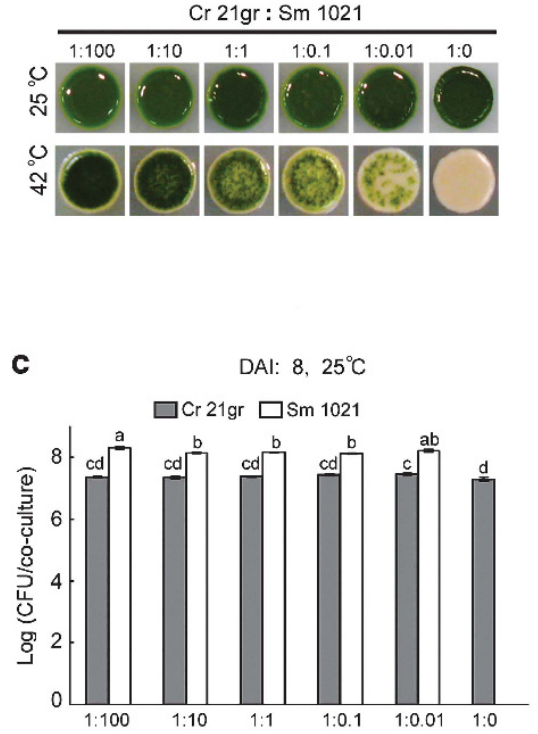

e

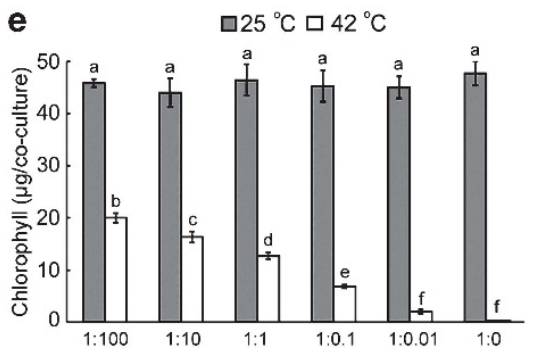

d

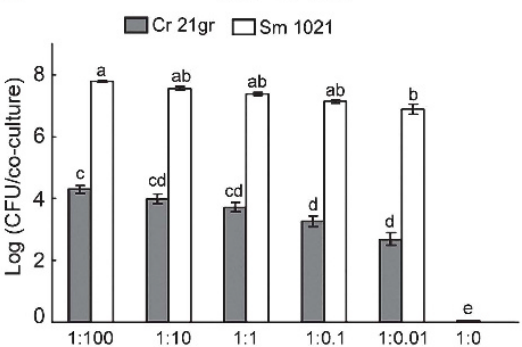

f

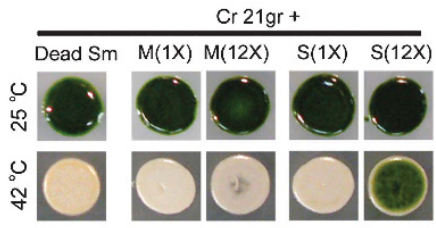

Figure 1 Enhancement of $C$. reinhardtii thermal tolerance by $S$. meliloti co-cultivation. (a) S. meliloti 1021 enhances C. reinhardtii 21 gr survival at lethal temperatures in a cell density-dependent manner using a co-culture thermal tolerance assay described in the Materials and methods and the footnote to Table 1. Photographs show the extent of chlorosis 5 days after the temperature upshift. (b-d) Viability of C. reinhardtii $21 \mathrm{gr}$ and $S$. meliloti 1021 in co-cultures incubated at $25^{\circ} \mathrm{C} 3$ (b) and 8 (c) days after inoculation (DAI) and at $42{ }^{\circ} \mathrm{C}$ for 5 days (d) after the temperature upshift (8 DAI). (e) Chlorophyll content of C. reinhardtii-S. meliloti co-cultures 8 DAI. (f) Co-inoculation of C. reinhardtii 21gr with dead S. meliloti (Sm) cells (1:100 Cr 21gr:Sm 1021), un-concentrated (1X) and concentrated 12-fold (12X) culture medium (M) or S. meliloti culture supernatant (S). Values are the mean \pm s.e.m. of three independent experiments each comprised of three replications. A separate one-way ANOVA was performed for C. reinhardtii 21gr and S. meliloti 1021 at each temperature and sampling time. Bars with different letters are significantly different based on a Duncan's test $(P \leqslant 0.05)$.

Table 2 Enhancement of C. reinhardtii 21gr thermal tolerance by exogenous supplements ${ }^{\mathrm{a}}$

\begin{tabular}{lccc}
\hline & $\begin{array}{c}\text { Thermal tolerance enhancing } \\
\text { effect }\end{array}$ & $\begin{array}{c}\text { Minimum enhancing concentration } \\
\left(\mathrm{mgl}^{-1}\right)\end{array}$ & $\begin{array}{c}\text { Maximum concentration tested } \\
\left(\mathrm{mg} \mathrm{l}^{-1}\right)\end{array}$ \\
\hline Cyanocobalamin & $2+$ & 0.0001 & 5 \\
Biotin & - & - & 0.5 \\
Thiamine & - & - & 0.16 \\
Methionine & \pm & - & 2000 \\
Folate & - & $20+0.04$ & 0.04 \\
Methionine + folate & $2+$ & $0.0001+20$ & $200+0.04$ \\
Cyanocobalamin + methionine & $2+$ & $1+2000$ & \\
\hline
\end{tabular}

Thermal tolerance enhancing (TE) effect is defined as no $(-)$, weak $( \pm)$, moderate $(+)$, or robust $(2+)$.

${ }^{a}$ Media were amended with various concentrations of supplements to identify those that were effective in enhancing Chlamydomonas thermal tolerance. Cultures were grown on solid media at $25^{\circ} \mathrm{C}$ for 3 days before shifting to $42{ }^{\circ} \mathrm{C}$ for 5 days after which results were recorded.

grown at higher temperatures. Collectively, these data suggest that better thermal tolerance at high cell densities may be a consequence of earlier entry into a stationary-phase physiological state.
The ability of cyanocobalamin and, to a lesser extent, methionine to enhance $C$. reinhardtii heat tolerance did not rely on surface growth as similar effects were attained in liquid culture (Figure 2d, 


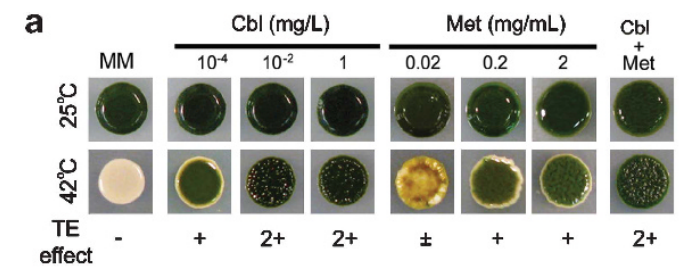

d
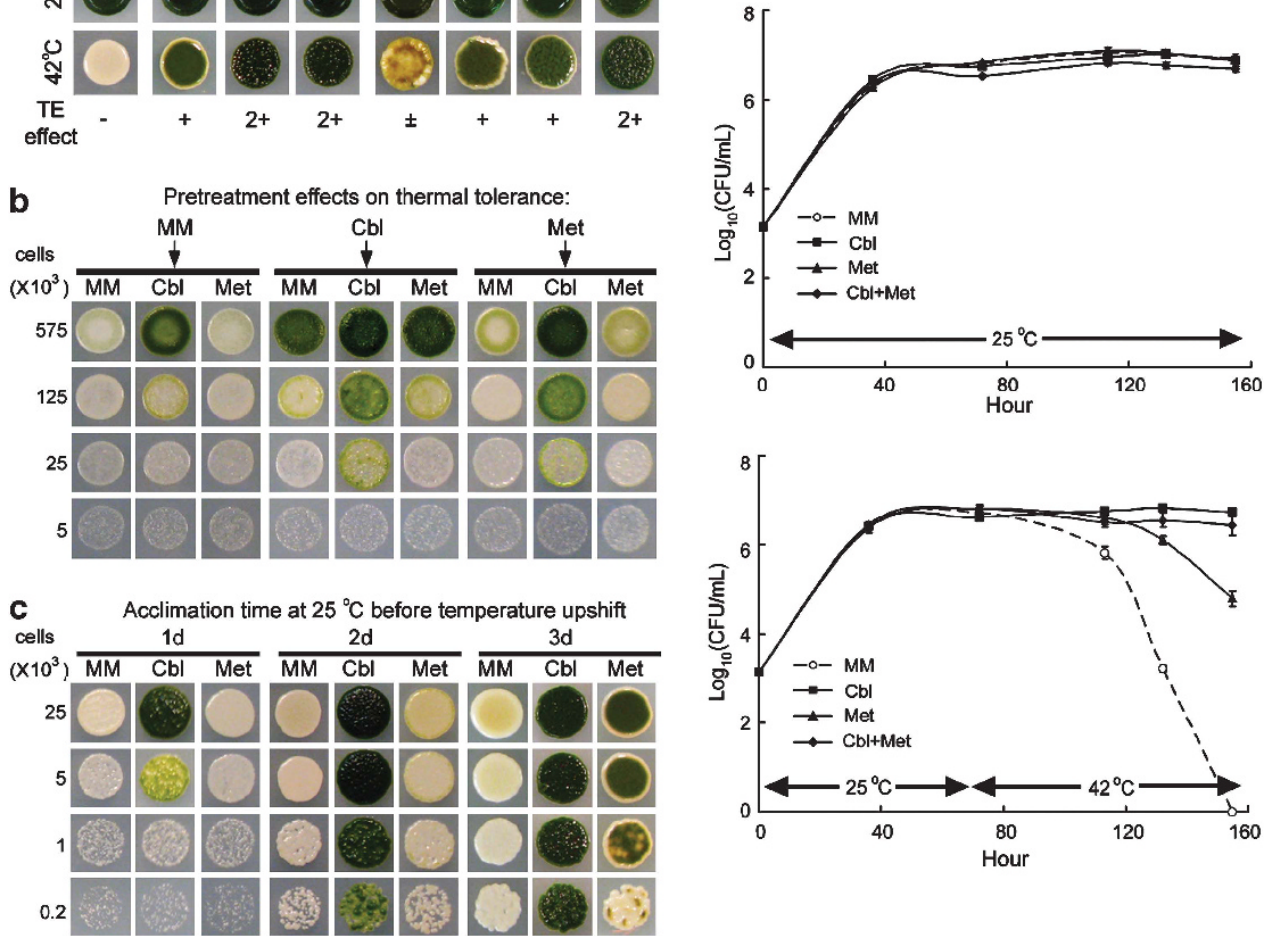

Figure 2 Enhancement of $C$. reinhardtii thermal tolerance by cyanocobalamin and methionine. (a) Thermal tolerance enhancing effect of C. reinhardtii 21gr on solid media un-amended (MM) or amended with cyanocobalamin (Cbl), methionine (Met) or both cyanocobalamin $\left(1 \mathrm{mgl}^{-1}\right)$ and methionine $\left(0.2 \mathrm{mg} \mathrm{ml}^{-1}\right)$, based on the thermal tolerance assay. Photographs were taken 8 DAI. Thermal tolerance enhancing effect (TE effect) is defined in Table 1. (b) Thermal tolerance of C. reinhardtii 21gr grown in the presence of cyanocobalamin $\left(1 \mathrm{mg} \mathrm{l}^{-1}\right)$ or methionine $\left(0.2 \mathrm{mg} \mathrm{ml}^{-1}\right)$ and in the absence of a 3-day acclimation period before the temperature upshift. Solid media was un-amended or amended with cyanocobalamin $\left(1 \mathrm{mgl}^{-1}\right)$ or methionine $\left(0.2 \mathrm{mg} \mathrm{ml}^{-1}\right)$. Photographs were taken 5 DAI. (c) Influence of acclimation time before the temperature upshift on thermal tolerance in the absence or presence of cyanocobalamin $\left(1 \mathrm{mg} \mathrm{l}^{-1}\right)$ or methionine $\left(0.2 \mathrm{mg} \mathrm{ml}^{-1}\right)$ for $1-3$ day before the temperature upshift. Photographs were taken 5 days after the temperature upshift. (d) Survival of $C$. reinhardtii $21 \mathrm{gr}$ in liquid culture without or with cyanocobalamin $\left(1 \mathrm{mg} \mathrm{l}^{-1}\right)$, methionine $\left(0.2 \mathrm{mg} \mathrm{ml}{ }^{-1}\right)$ or both amendments without (top) or with (bottom) $42^{\circ} \mathrm{C}$ temperature upshift. Values are the mean \pm s.e.m. of three independent experiments each comprised of five replications.

Supplementary Table S4), although viability of planktonic cells more quickly diminishes in the absence of cyanocobalamin or methionine following a temperature upshift (Figure 2d) compared with surface-grown cells (Figures 2a-c). A consequence of reduced viability at $42{ }^{\circ} \mathrm{C}$ in the absence of cyanocobalamin or methionine is a dramatic reduction in biomass compared with cyanocobalamin treatments (Supplementary Table S4), indicative of scavenging cellular resources to maintain viability.

S. meliloti produced adenosylcobalamin enhances

C. reinhardtii thermal tolerance

The preceding data indicated that cyanocobalamin mimics the effect of bacterial-mediated delay in chlorosis and prolonged survival of $C$. reinhardtii at lethal temperatures. To determine if bacterialmediated thermal tolerance was due to cobalamin production, we selected the $S$. meliloti cobalamindeficient $b l u B^{-}$and $c o b D^{-}$mutants to test this possibility. BluB synthesizes 5,6-dimethylbenzimidazole, the lower ligand of cobalamin while CobD is required for corrin ring synthesis (Campbell et al., 2006; Taga and Walker 2010). We also included a methionine auxotroph (metH $H^{-}$mutant) (Campbell et al., 2006; Taga and Walker, 2010). As expected, both the bluB $B^{-}$and $c o b D^{-}$mutants were dramatically impaired in delaying chlorosis or promoting C. reinhardtii survival (Figure 3). Differential survival of the $b l u B^{-}$and $c o b D^{-}$mutants is likely due to the presence of trace amounts of 5,6-dimethylbenzimidazole in the agar (Campbell et al., 2006), or $C$. reinhardtii exudates that facilitate $b l u B^{-}$ mutant survival (Figure 3c), but neither strain produces sufficient adenosylcobalamin to promote C. reinhardtii heat tolerance. Despite the poor survival of the metH $H^{-}$mutant in the absence of exogenous methionine (Figure 3c), sufficient amounts of cobalamin was produced to facilitate survival of Chlamydomonas at moderate levels. Collectively, these results suggest that cobalamin 

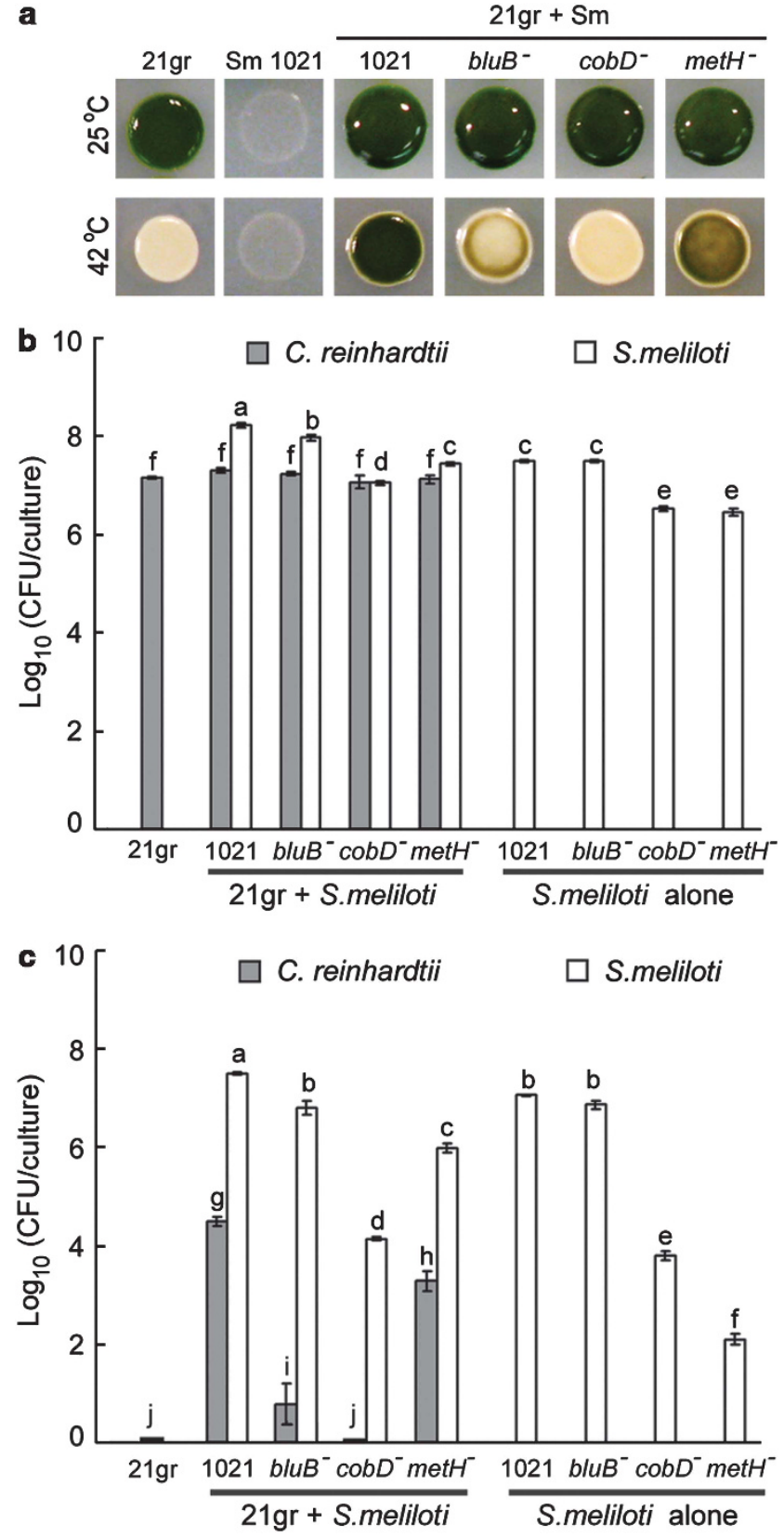

Figure 3 Adenosylcobalamin biosynthesis by $S$. meliloti confers thermal tolerance to $C$. reinhardtii. (a) Chlorosis of $C$. reinhardtii $21 \mathrm{gr}$ when cultivated with $S$. meliloti strain 1021 and various mutants deficient in adenosylcobalamin lower ligand $\left(b l u B^{-}\right)$or corrin ring $\left(\mathrm{cobD}^{-}\right)$synthesis, metH $H^{-}$in the thermal tolerance assay. Viability at $25^{\circ} \mathrm{C}(\mathbf{b})$ and $42{ }^{\circ} \mathrm{C}(\mathbf{c})$ was determined by plate counts 8 DAI. The Cr21gr:Sm 1021 inoculum ratio was 1:100 in all comparisons. Values represent the mean \pm s.e.m. of two independent experiments, each comprised of three replications. A separate ANOVA was performed for C. reinhardtii $21 \mathrm{gr}$ and S. meliloti 1021 and bars with different letters are significantly different based on a Duncan's test $(P \leqslant 0.05)$.

has a critical role in bacterial-mediated enhancement of $C$. reinhardtii thermal tolerance. Compared with growth at $25^{\circ} \mathrm{C}$, survival of $c o b D^{-}$and met $H^{-}$ mutants alone or in co-culture with $C$. reinhardtii were dramatically reduced at $42{ }^{\circ} \mathrm{C}$, implying that adenosylcobalamin and methionine production is also important for heat tolerance of S. meliloti.

This observation led us to investigate whether the inability of certain bacteria to enhance $C$. reinhardtii thermal tolerance was due to their inability to produce cobalamin. Many bacterial strains capable of aerobic cobalamin production were unable to enhance Chlamydomonas thermal tolerance (Table 1). Salmonella has served as a model for cobalamin synthesis (Raux et al., 1996; Roth et al., 1996; Warren et al., 2002), but only produces it under anaerobic conditions and it is unclear whether the other Enterobacteriaceae we tested also synthesize cobalamin anaerobically.

\section{Regulation of C. reinhardtii METE and METH by} temperature or bacteria

As exogenous cyanocobalamin and methionine rescues $C$. reinhardtii thermal sensitivity it suggests that either the expression or activity of the cobalamin-independent methionine synthase (METE) is heat-sensitive, whereas cobalamin-dependent $(M E T H)$ is not. We used quantitative RT-PCR to determine whether temperature, cyanocobalamin or S. meliloti 1021 co-culture affected METE and $M E T H$ expression. At a non-lethal $25^{\circ} \mathrm{C}$ temperature, C. reinhardtii METE is highly expressed (Figure 4a) but in the presence of cyanocobalamin METE is strongly repressed, whereas METH expression is unaffected, which is consistent with other studies (Croft et al., 2005; Helliwell et al., 2011). Importantly, following a 1 -h heat shock at $42{ }^{\circ} \mathrm{C}$, METE expression is reduced to $<10 \%$ of the control but METH expression decreases to about 30\% of the control (Figure 4a). As expected the heat shock treatment dramatically induces expression of the heat shock protein gene HSP90A (Schulz-Raffelt et al., 2007) (Supplementary Figure S3).

We also assessed C. reinhardtii METE and METH expression during its interaction with $S$. meliloti wild-type and the $b l u B^{-}$mutant 2 days after a temperature upshift. At $25^{\circ} \mathrm{C}$, no significant differences in $C$. reinhardtii METE expression could be detected without or with $S$. meliloti wild-type or blu $B^{-}$mutant co-cultivation (Figure $4 \mathrm{~b}$ ). After an upshift to $38^{\circ} \mathrm{C}$, a temperature near the maximum C. reinhardtii growth temperature, METE expression was greatly reduced in the presence of $S$. meliloti wild-type and the $b l u B^{-}$mutant, although the decrease was greater in the presence of the wildtype than the bluB $B^{-}$mutant. There was almost no METE detected following a $42^{\circ} \mathrm{C}$ upshift (Figure $4 \mathrm{~b}$ ). In contrast, following a 38 or $42{ }^{\circ} \mathrm{C}$ temperature upshift, METH expression was not significantly affected by the presence or absence of $S$. meliloti wild-type and the bluB $B^{-}$mutant (Figure 4b). These findings support conclusions inferred in another study (Kazamia et al., 2012) that C. reinhardtii METE expression can be repressed as a consequence of its interaction with a cobalamin-producing bacterial 

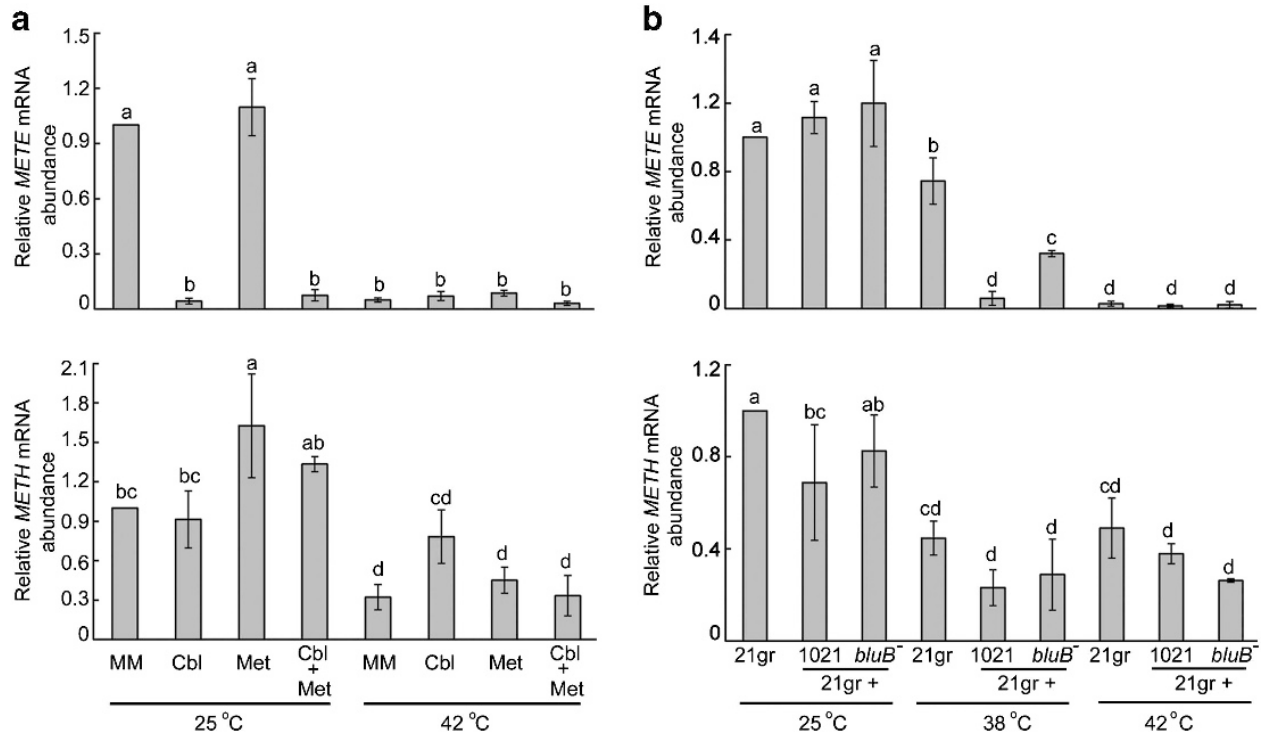

Figure 4 Quantitative real-time PCR of $C$. reinhardtii METE and METH mRNA. (a) $21 \mathrm{gr}$ cells were exposed to a 1 -h $42{ }^{\circ} \mathrm{C}$ heat shock or maintained at $25^{\circ} \mathrm{C}$ in the absence $(\mathrm{MM})$ or presence of cyanocobalamin ( $\mathrm{Cbl} ; 1 \mathrm{mgl}^{-1}$ ), methionine (Met; $0.2 \mathrm{mg} \mathrm{ml}{ }^{-1}$ ) or both amendments (Cbl + Met). (b) C. reinhardtii 21gr grown on solid media without or with S. meliloti wild-type 1021 or bluB $B^{-}$mutant. The 21gr:Sm inoculation ratio was $\sim 1: 100$. Co-cultures were exposed to a temperature upshift for 2 days before isolating RNA. The constitutively expressed CBLP gene (Schloss 1990) was used as the reference gene and values are normalized relative to 21gr cells grown at $25^{\circ} \mathrm{C}$ in $\mathrm{MM}$ medium. Bars are the mean \pm s.d. of two independent experiments. A separate ANOVA was performed for METE and $M E T H$ and bars with different letters are statistically different based on Duncan's test $(P \leqslant 0.05)$.
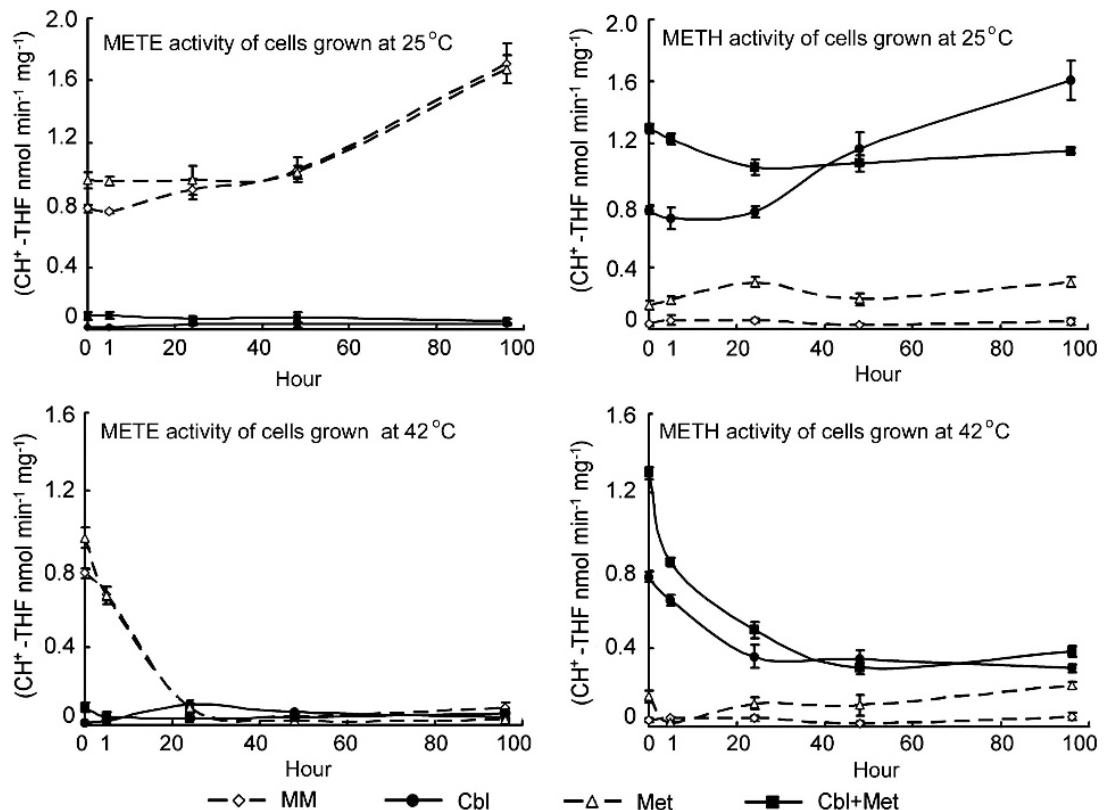

Figure 5 Effect of temperature on $C$. reinhardtii methionine synthase activity. METE and METH activity of $C$. reinhardtii cells grown in the absence $(\mathrm{MM})$ or presence of cyanocobalamin (Cbl; $1 \mathrm{mgl}^{-1}$ ), methionine (Met; $0.2 \mathrm{mg} \mathrm{ml}^{-1}$ ) or both amendments $(\mathrm{Cbl}+\mathrm{Met})$. Cultures were grown for 3 days at $25^{\circ} \mathrm{C}$ before a $42^{\circ} \mathrm{C}$ temperature upshift, except the controls were maintained at $25^{\circ} \mathrm{C}$. Crude cell extracts were used in the methionine synthase activity assays and all assays were conducted at $37^{\circ} \mathrm{C}$ for $45 \mathrm{~min}$, as described in the Materials and methods. Both assays measure the rate of methenyltetrahydrofolate $\left(\mathrm{CH}^{+}-\mathrm{THF}\right)$ production. Data are the mean \pm s.e.m. $(n=3)$.

species. The difference between these two studies is the requirement for a higher, non-lethal temperature (Figure 4b) and the supplementation of a bacterial carbon source in the study by Kazamia et al. (2012).

Our methionine synthase activity assays reflect the pool of enzymes isolated from $C$. reinhardtii cultures exposed to a temperature upshift for varying lengths of time (Figure 5). In both the METH and METE activity assays, the production of tetrahydrofolate was measured spectrophotometrically following its conversion to the more stable methenyltetrahydrofolate. METE activity was greatly 
reduced in the presence of a heat stress or cyanocobalamin, whereas during heat stress METH activity in the presence of cyanocobalamin was maintained at higher levels than METE (Figure 5). When not grown in the presence of cyanocobalamin and heat stress, METE activity was high, but heat stress caused a precipitous decline in activity. Following the temperature upshift, the METH activity of cyanocobalamin-treated $C$. reinhardtii decreased over time although these levels were substantially greater than METE activity (Figure 5); these levels may suffice to maintain adequate methionine pools to prolong survival.

\section{Gene silencing in C. reinhardtii}

To further our functional analysis of the role of METH in C. reinhardtii thermal tolerance, we took advantage of an amiRNA system to knock down METH expression. For this purpose a plasmid (pChlamiRNA3int-MetH) carrying the strong constitutive PSAD promoter (Molnar et al., 2009) was used to direct expression of an antisense cDNA amiRNA oligonucleotide targeting the $5^{\prime}$-end of $M E T H$ mRNA. Attempts to reduce $M E T H$ expression in strain 21gr transformed with this vector were unsuccessful based on screening 60 clones by qRTPCR and in the chlorosis assay. Five (Hi-1, $-2,-4,-12$, -21) of the 65 paromycin-resistant transformants of wild-type CC125 screened for reduction in METH expression by quantitative RT-PCR in 3-day-old cultures cultivated at a non-lethal $\left(25^{\circ} \mathrm{C}\right)$ temperature exhibited $10-50 \%$ of the METH expression of the empty vector control (Figure 6a). Furthermore, a reduction in METH expression in these five lines was highly correlated with a more rapid onset of chlorosis after temperature upshift even in the presence of cyanocobalamin, methionine or S. meliloti 1021 (Figure 6b). Low levels of METH expression in the five amiRNA lines had no apparent effect on growth and survival at $25^{\circ} \mathrm{C}$ in the absence of cobalamin (data not shown). Collectively these data provide strong evidence for the requirement of METH activity for the ability of cobalamin-producing bacteria to enhance $C$. reinhardtii thermal tolerance.

\section{Discussion}

Our findings indicate that interactions with cobalamin-producing bacteria can have profound effects on $C$. reinhardtii fitness as it responds to changes in environmental temperature and that methionine biosynthesis has a central role in thermal tolerance. This highlights the importance of the presence of cooccurring species in the acclimation of an organism to changes in its abiotic environment. Although concentrated culture supernatants delaying C. reinhardtii chlorosis at high temperature (Figure 1f) implies cobalamin could be scavenged from dead
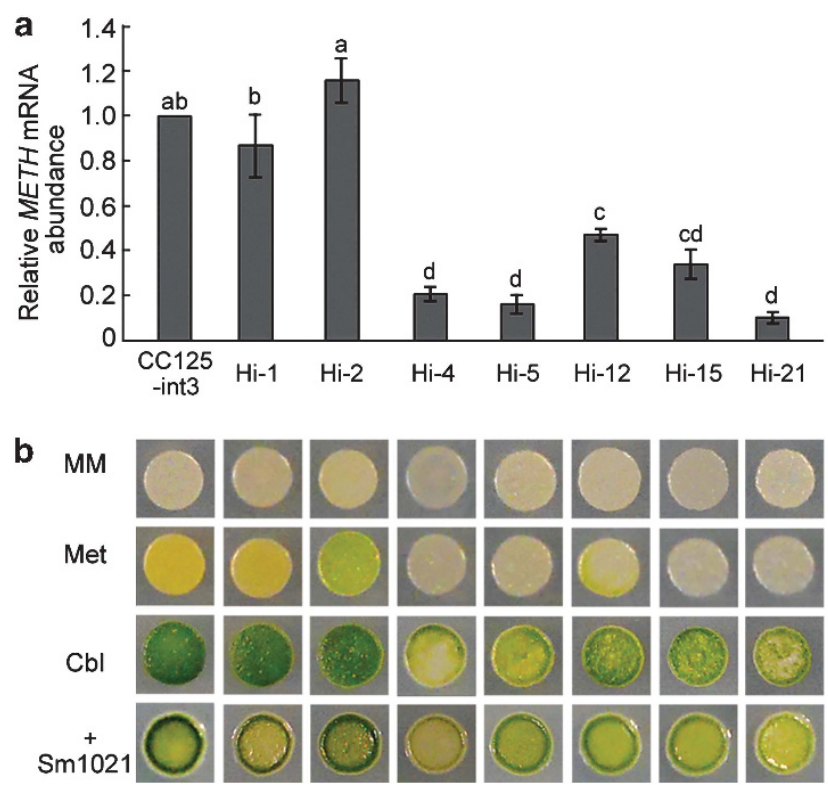

Figure 6 Phenotypes of $C$. reinhardtii lines expressing artificial microRNA (amiRNA) targeting METH mRNA. Quantitative realtime PCR (a) analysis of METH mRNA in METH-amiRNA expressing lines (Hi). Values are normalized relative to wild-type CC125 cells containing an empty pChlamiRNA3int vector (cc125int3) in 3-day-old broth cultures grown at $25^{\circ} \mathrm{C}$. Bars are the mean \pm s.e.m. $(n=3)$. Bars with different letters are statistically different based on a Duncan's test $(P \leqslant 0.05)$. Chlorosis phenotypes (b) of METH-amiRNA expressing cell lines following a temperature upshift in the presence of cyanocobalamin (Cbl; $1 \mathrm{mg} \mathrm{l}^{-1}$ ), methionine (Met; $0.2 \mathrm{mg} \mathrm{ml}^{-1}$ ) or S. meliloti 1021 (Sm 1021; Chlamydomonas: S. meliloti 1021 inoculum ratio of 1:100). Photographs were taken 8 DAI.

bacteria (Droop, 2007), several lines of evidence suggest otherwise. First, not all cobalamin-producing bacteria are capable of enhancing $C$. reinhardtii thermal tolerance (Table 1) and second, cobalamin needs to be present for a sufficient length of time before the temperature upshift to be functional (Figures $2 \mathrm{~b}$ and $\mathrm{c}$ ). In addition, cobalamin production is regulated by environmental conditions such as oxygen in a species-dependent manner (Jeter et al., 1984). Why some bacteria capable of aerobic cobalamin production enhance $C$. reinhardtii thermal tolerance and others do not is unclear, but conceivably it's a consequence of differences in bacterial thermal tolerance or ability to utilize C. reinhardtii resources that support bacterial growth under elevated temperatures. Bacteria we examined that strongly enhance $C$. reinhardtii thermal tolerance are members of the $\alpha$-proteobacterial class, which includes the Rhizobiales order. This is consistent with other reports (Hold et al., 2001; Sapp et al., 2007) of specific bacterial lineages, including $\alpha$-proteobacteria, being associated with various algal species in natural environments. These associations may reflect a certain degree of specificity, possibly a consequence of the suitability of photosynthate algae provide to heterotrophic bacteria (Kazamia et al., 2012), that is, there may be a degree of specificity in this chemical dependency. 
The ecology of bacterial-algal interactions is not well understood, but recent studies have highlighted how each partner can influence the physiology of the other. For example, bacterial promotion of algal assimilation of iron in exchange for photosynthate in marine systems (Amin et al., 2009) and C. reinhardtii secretion of substances mimicking the activity of $N$-acyl-L-homoserine lactone signal molecules used by $S$. meliloti and other soil bacteria for quorum-sensing regulation of gene expression (Teplitski et al., 2004; Rajamani et al., 2008). None of the $S$. meliloti proteins differently regulated by C. reinhardtii mimics were involved in adenosylcobalamin biosynthesis (Teplitski et al., 2004). Repression of $C$. reinhardtii METE expression at non-lethal elevated temperatures (Figure $4 \mathrm{~b}$ ) by adenosylcobalamin-producing $S$. meliloti provides direct evidence that algae gene expression can be influenced by cobalamin-producing bacteria. At present, it is unclear whether the greater METE repression at higher temperatures is a consequence of more adenosylcobalamin production by $S$. meliloti, more adenosylcobalamin release into the environment by $S$. meliloti cell death or more efficient uptake by C. reinharditii cells. Diatoms increase cobalamin acquisition, reduce cobalamin demand or mitigate cellular damage caused by reduced methionine biosynthesis capabilities under low cobalamin conditions (Bertrand et al., 2012). At present, we do not know whether similar strategies are employed by C. reinhardtii or other chlorophyta to alleviate cobalamin scarcity under elevated temperatures or whether they stimulate closer associations with cobalamin-producing bacteria. The minimum cobalamin concentration (74 pMol) required for enhancing $C$. reinhardtii thermal tolerance is greater than the concentration reported in the few studies examining natural cobalamin concentrations in aquatic and terrestrial environments (Schneider and Stroinski, 1987; Panzeca et al., 2009; SañudoWilhelmy et al., 2012), and greater than that required to support growth of algal vitamin $B_{12}$ auxotrophs (Croft et al., 2005, Kazamia et al., 2012). Thus, in environments depleted in cobalamin, such as those along the southern California-Baja California coastal ocean (Sañudo-Wilhelmy et al., 2012), alga cobalamin auxotrophs would be scarce or algae would be more efficient in cobalamin acquisition or rely more on closer associations with cobalaminproducing bacteria.

Methionine is central to $C$. reinhardtii thermal tolerance, given that as little as a $50 \%$ reduction in METH expression incapacitates cobalaminmediated enhancement of thermal tolerance (Figure 6). Methionine is not only required for protein synthesis but also for methylation reactions and methyl cycling, while methionine deficiencies can lead to accumulation of homocysteine to toxic levels. Heat repression of $C$. reinhardtii METE gene expression could explain decreases in METE pools during heat stress (Figures 4 and 5). In contrast expression of METH was less affected by heat shock, (Figure 4) which likely contributes to the presence of, albeit small, pool sizes of METH during heat stress (Figure 5) over prolonged heat exposure. The effect of heat on C. reinhardtii METE gene expression and METE pools is consistent with those reported for other organisms, including C. reinhardtii (Muhlhaus et al., 2011); it is conceivable that METH is also heat-labile although to a lesser extent than METE. In E. coli, MetE protein is susceptible to heat-induced aggregation and degradation (Mogk et al., 1999), which stimulates the heat shock responses. Thus, C. reinhardtii methionine pools and biosynthesis proteins may be central to growth rate control at elevated temperatures and in activating the heat shock response (Ron and Davis, 1971; Ron 1975; Gur et al., 2002) as well as for maintaining de novo protein biosynthesis capabilities required for photosynthetic machinery acclimation to higher temperatures (Tanaka et al., 2000).

The fitness advantage to $C$. reinhardtii of the cobalamin-dependent (METH) over the cobalaminindependent (METE) methionine synthase might help explain the retention of $M E T H$ gene and the apparent independent loss of METE gene in various algal species. A recent survey of cobalamin-dependent enzymes in 15 sequenced algal genomes representative of the Chlorphyta, Chromalveolata and Rhodophyta highlighted the nearly ubiquitous presence of the METH gene, whereas the METE gene was detected in only $50 \%$ of the genomes (Helliwell et al., 2011). Organisms with both METE and METH are clearly more flexible in environments that routinely or periodically lack cobalamin availability, but the greater thermal tolerance properties of METH provides for greater plasticity to address changes in environmental temperature despite the cobalamin chemical dependency.

Our findings also have implications for understanding ecosystem responses to global temperature changes. Following an increase in environmental temperature, we could anticipate changes in microbial community composition, including more cobalamin-producers, more efficient cobalaminscavengers or variants with physiological adaptations circumventing methionine limitations or with more heat tolerant cobalamin-independent methionine synthases (METE). As a consequence of shifts in microbial community structure or metabolic activities, primary productivity, a key component of the global carbon cycle, could be disrupted in the absence of sufficient abundance of cobalamin or cobalamin-producing bacteria. Thus, it might be appropriate to include cobalamin availability and cobalamin-producing bacteria and their production and consumption dynamics in efforts to predict and model marine or terrestrial primary productivity as temperatures rise. Our studies also suggest that optimal algal productivity in engineered systems could be more consistently achieved by relying on cobalamin-dependent (METH) rather than 
cobalamin-independent (METE) methionine synthase in systems that experience operating temperatures that cause heat stress to algae, thereby decreasing productivity. Consequently, production systems could be operated at higher temperatures in the presence of cobalamin, thereby reducing cooling costs.

\section{Acknowledgements}

This research was supported by the Department of Energy Advanced Research Program Agency-Energy (DE-AR0000010) and by the Iowa Agriculture and Home Economics Experiment Station. We thank Dr Yingjun Wang and Michael Rand for technical assistance, and Dr Zonglie Hong for helpful suggestions.

\section{References}

Amin SA, Green DH, Hart MC, Küpper FC, Sunda WG, Carrano CJ. (2009). Photolysis of iron-siderophore chelates promotes bacterial-algal mutualism. Proc Natl Acad Sci USA 106: 17071-17076.

Barott KL, Rodriguez-Brito B, Janouskovec J, Marhaver KL, Smith JE, Keeling P et al. (2011). Microbial diversity associated with four functional groups of benthic reef algae and the reef-building coral Montastraea annularis. Environ Microbiol 13: 1192-1204.

Beringer JE. (1974). $\mathrm{R}$ factor transfer in Rhizobium leguminosarum. J Gen Microbiol 84: 188-198.

Bertrand EM, Allen AE, Dupont CL, Norden-Krichmar TM, Bai J, Valas RE et al. (2012). Influence of cobalamin scarcity on diatom molecular physiology and identification of a cobalamin acquisition protein. Proc Natl Acad Sci USA 109: E1762-E1771.

Broughton WJ, Wong CH, Lewin A, Samrey U, Myint H, Meyer $\mathrm{H}$ et al. (1986). Identification of Rhizobium plasmid sequences involved in recognition of Psophocarpus, Vigna, and other legumes. J Cell Biol 102: 1173-1182.

Campbell GR, Taga ME, Mistry K, Lloret J, Anderson PJ, Roth JR et al. (2006). Sinorhizobium meliloti bluB is necessary for production of 5,6-dimethylbenzimidazole, the lower ligand of B12. Proc Natl Acad Sci USA 103: 4634-4639.

Croft MT, Lawrence AD, Raux-Deery E, Warren MJ, Smith AG. (2005). Algae acquire vitamin B12 through a symbiotic relationship with bacteria. Nature 438: 90-93.

Croft MT, Warren MJ, Smith AG. (2006). Algae need their vitamins. Eukaryot Cell 5: 1175-1183.

Droop MR. (2007). Vitamins, phytoplankton and bacteria: symbiosis or scavenging? J Plankton Res 29: 107-113.

Drummond JT, Jarrett J, Gonzalez JC, Huang S, Matthews RG. (1995). Characterization of nonradioactive assays for cobalamin-dependent and cobalamin-independent methionine synthase enzymes. Anal Biochem 228: 323-329.

Falkowski PG, Katz ME, Knoll AH, Quigg A, Raven JA, Schofield O et al. (2004). The evolution of modern eukaryotic phytoplankton. Science 305: 354-360.

Field CB, Behrenfeld MJ, Randerson JT, Falkowski P. (1998). Primary production of the biosphere: integrating terrestrial and oceanic components. Science 281: $237-240$
Geraghty AM, Anderson JC, Spalding MH. (1990). A 36 kilodalton limiting-CO(2) induced polypeptide of Chlamydomonas is distinct from the 37 kilodalton periplasmic carbonic anhydrase. Plant Physiol 93: 116-121.

Gonzalez JC, Banerjee RV, Huang S, Sumner JS, Matthews RG. (1992). Comparison of cobalamin-independent and cobalamin-dependent methionine synthases from Escherichia coli: two solutions to the same chemical problem. Biochemistry 31: 6045-6056.

Gorman DS, Levine RP. (1965). Cytochrome $f$ and plastocyanin: their sequence in the photosynthetic electron transport chain of Chlamydomonas reinhardii. Proc Natl Acad Sci USA 54: 1665-1669.

Gur E, Biran D, Gazit E, Ron EZ. (2002). In vivo aggregation of a single enzyme limits growth of Escherichia coli at elevated temperatures. Mol Microbiol 46: 1391-1397.

Helliwell KE, Wheeler GL, Leptos KC, Goldstein RE, Smith AG. (2011). Insights into the evolution of vitamin B12 auxotrophy from sequenced algal genomes. Mol Biol Evol 28: 2921-2933.

Hold GL, Smith EA, Rappé MS, Maas EW, Moore ERB, Stroempl C et al. (2001). Characterisation of bacterial communities associated with toxic and non-toxic dinoflagellates: Alexandrium spp. and Scrippsiella trochoidea. FEMS Microbio Eco 37: 161-173.

Jeter RM, Olivera BM, Roth JR. (1984). Salmonella typhimurium synthesizes cobalamin (vitamin B12) de novo under anaerobic growth conditions. J Bacteriol 159: 206-213.

Kazamia E, Czesnick H, Nguyen TTV, Croft MT, Sherwood E, Sasso S et al. (2012). Mutualistic interactions between vitamin B12-dependent algae and heterotrophic bacteria exhibit regulation. Environ Microbiol 14: 1466-1476.

Kirk DL, Kirk MM. (1978). Carrier-mediated uptake of arginine and urea by Chlamydomonas reinhardtii. Plant Physiol 61: 556-560.

Matsuo Y, Imagawa H, Nishizawa M, Shizuri Y. (2005). Isolation of an algal morphogenesis inducer from a marine bacterium. Science 307: 1598.

Matz C, Webb JS, Schupp PJ, Phang SY, Penesyan A, Egan S et al. (2008). Marine biofilm bacteria evade eukaryotic predation by targeted chemical defense. PLOS ONE 3: e2744.

Mogk A, Tomoyasu T, Goloubinoff P, Rudiger S, Roder D, Langen $\mathrm{H}$ et al. (1999). Identification of thermolabile Escherichia coli proteins: prevention and reversion of aggregation by DnaK and ClpB. EMBO J 18: 6934-6949.

Molnar A, Bassett A, Thuenemann E, Schwach F, Karkare S, Ossowski S et al. (2009). Highly specific gene silencing by artificial microRNAs in the unicellular alga Chlamydomonas reinhardtii. Plant J (2009). 58: 165-174.

Muhlhaus T, Weiss J, Hemme D, Sommer F, Schroda M. (2011). Quantitative shotgun proteomics using a uniform (1)N-labeled standard to monitor proteome dynamics in time course experiments reveals new insights into the heat stress response of Chlamydomonas reinhardtii. Mol Cell Proteomics 10: 004739.

Panzeca C, Beck AJ, Tovar-Sanchez A, Segovia-Zavala J, Taylor GT, Gobler CJ et al. (2009). Distributions of dissolved vitamin B12 and Co in coastal and openocean environments. Est Coast Shelf Sci 85: 223-230.

Rajamani S, Bauer WD, Robinson JB, Farrow JM, Pesci EC, Teplitski $\mathrm{M}$ et al. (2008). The vitamin riboflavin and its derivative lumichrome activate the LasR bacterial quorum-sensing receptor. Mol Plant Microb Interact 21: 1184-1192. 
Raux E, Lanois A, Levillayer F, Warren MJ, Brody E, Rambach A et al. (1996). Salmonella typhimurium cobalamin (vitamin B12) biosynthetic genes: functional studies in S. typhimurium and Escherichia coli. J Bacteriol 178: 753-767.

Ron EZ. (1975). Growth rate of Enterobacteriaceae at elevated temperatures: limitation by methionine. J Bacteriol 124: 243-246.

Ron EZ, Davis BD. (1971). Growth rate of Escherichia coli at elevated temperatures: limitation by methionine. J Bacteriol 107: 391-396.

Roth JR, Lawrence JG, Bobik TA. (1996). Cobalamin (coenzyme B12): synthesis and biological significance. Annu Rev Microbiol 50: 137-181.

Sapp M, Schwaderer A, Wiltshire K, Hoppe H-G, Gerdts G, Wichels A. (2007). Species-specific bacterial communities in the phycosphere of microalgae? Microbial Ecol 53: 683-699.

Sañudo-Wilhelmy SA, Cutter LS, Durazo R, Smail EA, Gómez-Consarnau L, Webb EA et al. (2012). Multiple B-vitamin depletion in large areas of the coastal ocean. Proc Natl Acad Sci USA 109: 14041-14045.

Schloss JA. (1990). A Chlamydomonas gene encodes a G protein $\beta$ subunit-like polypeptide. Mol Gen Genet 221: $443-452$.

Schneider Z, Stroinski A. (1987). The occurrence and distribution of corrinoids. Comprehensive B12: Chemistry, Biochemistry, Nutrition, Ecology, Medicine. Walter de Gruyter \& Co.: Berlin, Germany, pp 157-224.
Schroda M. (2004). The Chlamydomonas genome reveals its secrets: chaperone genes and the potential roles of their gene products in the chloroplast. Photosynth Res 82: 221-240.

Schulz-Raffelt M, Lodha M, Schroda M. (2007). Heat shock factor 1 is a key regulator of the stress response in Chlamydomonas. Plant J 52: 286-295.

Seyedsayamdost MR, Carr G, Kolter R, Clardy J. (2011). Roseobacticides: Small molecule modulators of an algalbacterial symbiosis. J Amer Chem Soc 133: 18343-18349.

Taga ME, Larsen NA, Howard-Jones AR, Walsh CT, Walker GC. (2007). BluB cannibalizes flavin to form the lower ligand of vitamin B12. Nature 446: 449-453.

Taga ME, Walker GC. (2010). Sinorhizobium meliloti requires a cobalamin-dependent ribonucleotide reductase for symbiosis with its plant host. Mol Plant Microbe Interact 23: 1643-1654.

Tanaka Y, Nishiyama Y, Murata N. (2000). Acclimation of the photosynthetic machinery to high temperature in Chlamydomonas reinhardtii requires synthesis de novo of proteins encoded by the nuclear and chloroplast genomes. Plant Physiol 124: 441-449.

Teplitski M, Chen H, Rajamani S, Gao M, Merighi M, Sayre RT et al. (2004). Chlamydomonas reinhardtii secretes compounds that mimic bacterial signals and interfere with quorum sensing regulation in bacteria. Plant Physiol 134: 137-146.

Warren MJ, Raux E, Schubert HL, Escalante-Semerena JC. (2002). The biosynthesis of adenosylcobalamin (vitamin B12). Nat Prod Rep 19: 390-412.

Supplementary Information accompanies this paper on The ISME Journal website (http://www.nature.com/ismej) 